\title{
Correspondence
}

\section{Induction of autophagy does not alter the anti-tumor effects of HDAC inhibitors}

\author{
A Newbold ${ }^{1,3}$, SJ Vervoort ${ }^{1,3,4}$, BP Martin ${ }^{1}$, M Bots $^{\star, 1,5}$ and RW Johnstone ${ }^{\star, 1,2,5}$ \\ Cell Death and Disease (2012) 3, e387; doi:10.1038/cddis.2012.128; published online 6 September 2012 \\ Subject Category: Cancer
}

Dear Editor,

HDAC inhibitors (HDACis) can suppress the growth and/or survival of tumor cells and thus far two HDACis, vorinostat and romidepsin, have been approved by the FDA for the treatment of hematological malignancies. ${ }^{1}$ Vorinostat is a potent $\mathrm{HDACi}$ that mediates tumor cell-selective apoptosis and we and others have previously shown that the induction of apoptosis correlates with therapeutic efficacy in mouse models of hematological cancer. ${ }^{2-5}$ Although apoptosis may be the preferred mode of HDACi-induced cell death, it is clear that in tumor cells with nonfunctional apoptotic cascades, HDACi are capable of inducing a caspase-independent form of cell death. ${ }^{5-7}$ We recently demonstrated that $\mathrm{E} \mu$-myc lymphomas devoid of Apaf- 1 displayed a delayed cell death in response to HDACi treatment, and loss of Apaf-1 failed to affect therapeutic efficacy. ${ }^{5}$ The delayed HDACi-induced cell death was concomitant with biochemical and morphological changes characteristic of autophagy. ${ }^{5}$ Herein we utilized the genetically tractable $\mathrm{E} \mu-m y c$ mouse model to address conflicting reports in the field regarding the importance of autophagy in regulating the anti-tumor responses of $\mathrm{HDACi}^{6-8}$

To determine whether autophagy had any role in regulating

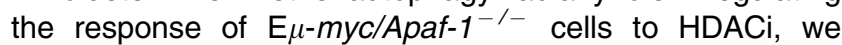
knocked down the expression of two key autophagy proteins (Atg5 and Atg7) using constitutive short-hairpin RNAs (shRNAs). We developed two distinct shRNAs against Atg5 and one shRNA against Atg7 that efficiently silenced the expression of Atg5 and Atg7, respectively (Figure 1a). To determine if knockdown of Atg5 or Atg7 impaired HDACimediated autophagy, we treated Atg5- and Atg7-shRNAexpressing $\mathrm{E} \mu$-myc/Apaf-1 ${ }^{-/-}$lymphomas with vorinostat and determined the ratio of LC3-I/LC3-II by western blot. In $\mathrm{E} \mu-\mathrm{myc} / \mathrm{Apaf}^{-1^{-/-}}$cells with decreased expression of Atg5 or Atg7, vorinostat-induced processing of LC3-I to LC3-II was greatly reduced (Figure 1a).
We next evaluated the response of Atg5- and Atg7-shRNAexpressing $\mathrm{E} \mu-\mathrm{myc} / \mathrm{Apaf}^{-1^{-/}}$lymphomas to increasing concentrations of vorinostat over time. Consistent with our previous results, ${ }^{5}$ a delay in the kinetics of cell death was observed in vorinostat-treated $\mathrm{E} \mu-m y c /$ Apaf- $^{-1 /}$ compared with $\mathrm{E}_{\mu}$-myc lymphomas (Figure 1b). However, depletion of Atg5 or Atg7 did not affect vorinostat-mediated tumor cell death, indicating that inhibition of autophagy neither protected E $\mu$-myc/Apaf-1 ${ }^{-/-}$cells from HDACi-mediated cell death nor potentiated its anticancer effect. To evaluate the response of Atg5- and Atg7-shRNA-expressing E $\mu$-myc/Apaf-1 ${ }^{-1-}$ lymphomas to vorinostat in vivo, we transplanted lymphomas into recipient mice and treated tumor-bearing mice with vorinostat. Consistent with our in vitro data, Atg5- and Atg7-shRNA-expressing lymphomas were equally responsive to vorinostat and cleared with similar kinetics as controlshRNA-expressing lymphomas (Figure 1c). Finally, we determined whether inhibition of autophagy had any effect on the therapeutic efficacy of vorinostat in $\mathrm{E} \mu$-myc/Apaf-1 ${ }^{-/-}$ lymphomas. We observed an enhanced survival in mice bearing Atg5-shRNA E $\mu$-myc/Apaf-1 ${ }^{-/-}$lymphomas after treatment with vorinostat (median survival vorinostat: 43.5 days, median survival vehicle: 25 days, $P<0.0084$ ), which was similar to the therapeutic response seen with controlshRNA-expressing lymphomas (median survival vorinostat: 48 days compared with vehicle: 23.5 days, $P<0.0001$ ). In conclusion, our data demonstrate that there is no evidence supporting an essential role for autophagy in regulating the response of apoptosis-deficient tumors to HDACi. Recent data suggest that in tumor cells with apoptotic defects, inhibition of autophagy may potentiate the therapeutic response mediated by $\mathrm{HDACi}^{7}$ In contrast, we provide evidence that combining autophagy inhibitors with $\mathrm{HDACi}$ may not be clinically beneficial in lymphomas with apoptotic defects.

\footnotetext{
${ }^{1}$ Cancer Therapeutics Program, Peter MacCallum Cancer Centre, St. Andrews Place, East Melbourne 3002, Victoria, Australia and ${ }^{2}$ Sir Peter MacCallum Department of Oncology, University of Melbourne, Parkville 3010, Victoria, Australia

*Corresponding authors: M Bots. Current address: Laboratory for Experimental Oncology and Radiobiology (LEXOR), Center for Experimental Molecular Medicine, Academic Medical Center, Amsterdam, The Netherlands. Tel: +31 20 5664824; Fax: +31 20 6977192; E-mail: m.bots@amc.uva.nl or RW Johnstone, Cancer Therapeutics Program, Peter MacCallum Cancer Centre, St. Andrews Place, East Melbourne 3002, Victoria, Australia. Tel: +61 396563727 ; Fax: +61 39656 1411; E-mail: ricky.johnstone@ petermac.org

${ }^{3}$ Co-first authors.

${ }^{4}$ Current address: Department of Cell Biology, University Medical Center Utrecht, Utrecht, The Netherlands.

${ }^{5}$ Co-senior authors.
} 


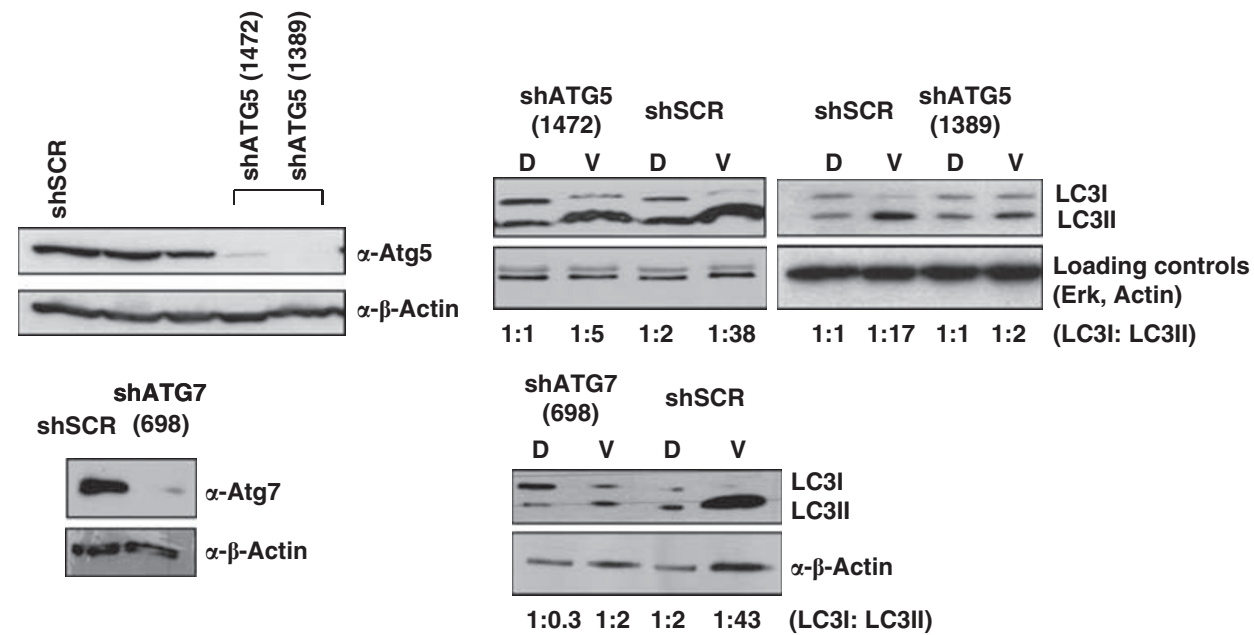

b
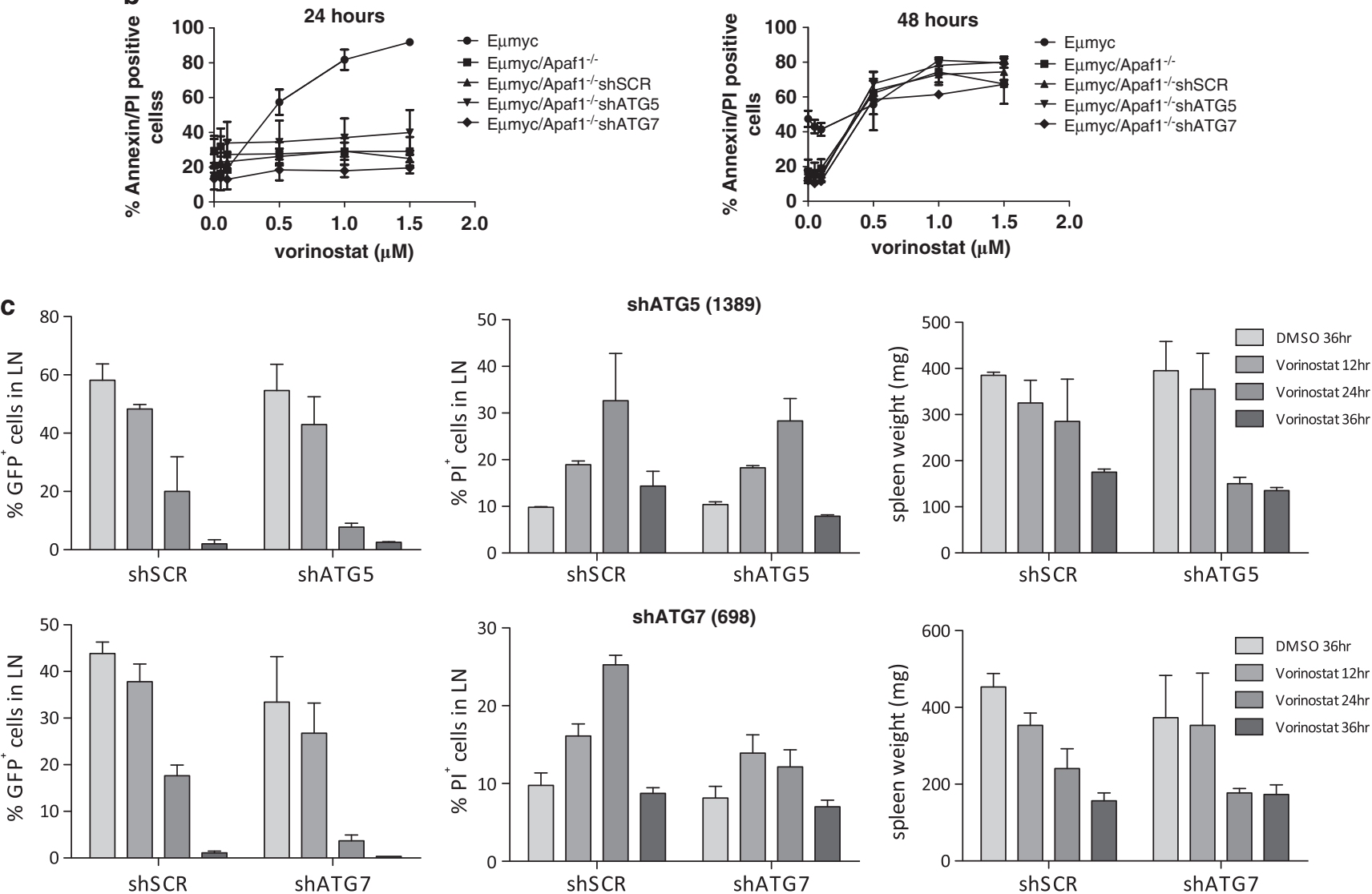

Figure 1 Vorinostat does not require a functional autophagic pathway to induce tumor cell death in $\mathrm{E} \mu$-myc/Apaf-1 ${ }^{-/-}$lymphomas. (a) $\mathrm{E} \mu-m y c / A p a f-1^{-/-}$lymphomas were retrovirally transduced with constructs expressing shRNAs targeting Atg5 (clone 1472 and 1389, respectively), Atg7 (clone 698) or a scrambled control. Knockdown efficiency was tested via western blot using antibodies specific for Atg5 and Atg7 (Cell Signaling Technologies, Inc., Danvers, MA, USA, no. 2630 and 2631). Anti- $\beta$-actin was used as a loading control. Results are a representative of at least three separate westerns. Atg5-shRNA-expressing E $\mu$-myc/Apaf-1 ${ }^{-/-}$(1389 and 1472), Atg7-shRNAexpressing $\mathrm{E} \mu$-myc/Apaf-1-1- (698) and control-shRNA-expressing $\mathrm{E} \mu$-myc/Apaf-1 ${ }^{-1-}$ lymphomas were treated with $1 \mu \mathrm{M}$ vorinostat (V) or DMSO (D) for $24 \mathrm{~h}$ and LC3 processing was determined by western blot using antibody specific for LC3 (NanoTools Antikoerpertechnik GmbH \& Co. KG., Teningen, Germany, no. 0260-100/LC3-2G6). Anti-p44/42 ERK (Cell Signaling Technology, Inc., Danvers, MA, USA, no. 9107) or anti- $\beta$-actin were used as loading controls. Quantitative western analysis was performed using ImageJ software (public domain software by Wayne Rasband), National Institute of Health, USA. Results are representative of at least three separate westerns. (b) $\mathrm{E} \mu-m y c, \mathrm{E} \mu-\mathrm{myc} / \mathrm{Apaf}-1^{-/-}$and $\mathrm{E} \mu-\mathrm{myc} / \mathrm{Apaf}-1^{-/-}$lymphomas expressing the various shRNA constructs were treated with increasing concentrations of vorinostat for 24 and $48 \mathrm{~h}$. Cell viability was assessed by annexin V/propidium iodide staining and error bars indicate \pm S.E.M. of at least three independent experiments. (c) C57B//6 mice bearing Atg5-shRNA-expressing E $\mu$-myc/Apaf-1 ${ }^{-/-}$(1389), Atg7-shRNA-expressing E $\mu$-myc/Apaf-1 ${ }^{-/-}$(698) or control-shRNA-expressing E $\mu$-myc/Apaf-1 ${ }^{-/-}$ lymphomas were treated with one dose of vorinostat $(200 \mathrm{mg} / \mathrm{kg}$ ). Spleen and lymph nodes were harvested at the indicated time points (h) following HDACi treatment and the percentage of lymphoma cells in lymph nodes was determined using flow cytometry in the presence of propidium iodide. Each time point is representative of 2-3 mice 


\section{Conflict of Interest}

The authors declare no conflict of interest.

Acknowledgements. MB was supported by the Dutch Cancer Society (AMC2009-4457). RWJ is a Principal Research Fellow of the National Health and Medical Research Council of Australia (NHMRC) and supported by NHMRC Program and Project Grants, the Susan G Komen Breast Cancer Foundation, Cancer Council Victoria, The Victorian Cancer Agency, The Leukemia Foundation of Australia and the Victorian Breast Cancer Research Consortium.

1. Bolden JE, Peart MJ, Johnstone RW. Nat Rev Drug Discov 2006; 5: 769-784. 2. Nebbioso A et al. Nat Med 2005; 11: 77-84.
3. Lindemann RK et al. Proc Natl Acad Sci USA 2007; 104: 8071-8076.

4. Newbold A et al. Mol Cancer Ther 2008; 7: 1066-1079.

5. Ellis $L$ et al. Blood 2009; 114: 380-393.

6. Shao Y et al. Proc Natl Acad Sci USA 2004; 101: 18030-18035.

7. Gammoh N et al. Proc Natl Acad Sci USA 2012; 109: 6561-6565.

8. Liu YL et al. Autophagy 2010; 6: 1057-1065.

Cell Death and Disease is an open-access journal published by Nature Publishing Group. This work is licensed under the Creative Commons Attribution-NonCommercial-No Derivative Works 3.0 Unported License. To view a copy of this license, visit http://creativecommons.org/licenses/by-nc-nd/3.0/ 